\title{
Comparative analysis of functional outcome for CT-based versus MRI-based evaluation in acute ischemic stroke prior to mechanical thrombectomy
}

\author{
Atulabh Vajpeyee ${ }^{1 *} \mathbb{D}$, Shivam Tiwari ${ }^{1 \dagger}$, Lokendra Bahadur Yadav ${ }^{1}$, Narendra Mal ${ }^{2}$, Kapil Vyas $^{3}$, \\ Dan Neftalie Juangco ${ }^{1}$, Sigit Dewanto Hendrani ${ }^{1}$ and Manisha Vajpeyee ${ }^{1,4}$
}

\begin{abstract}
Background: This study aims to compare functional outcome for Computed tomography (CT)-based versus Magnetic resonance imaging (MRI)-based evaluation in acute ischemic stroke patients prior to Mechanical thrombectomy (MT) in less than 6-h window period in anterior circulation stroke. Participants were patients admitted from September 2, 2018 to September 2, 2020 with an acute ischemic stroke who underwent mechanical thrombectomy treatment. Total duration of MRI stroke protocol and CT scan with Computed tomography angiography (CTA) was 12 min $57 \mathrm{~s}$, and 9 min $23 \mathrm{~s}$, respectively. Follow-up for periodic Modified Rankin Scale (MRS) was performed at 3 months.

Results: Number of patients included in the study were 152 with mean age of 54.6 (range 22-80) years with male predominance $(n=102)$. Mean GCS on admission was 12 (4-15) and 13(4-14) in CT and MRI group, respectively. National Institute of Health stroke scale (NIHSS) on admission was 17 (4-30) and 16(4-30) and at discharge was 7 (2-23) and 6(2-22) in CT-based group and MRI-based group, respectively. In the MRI group 65.5\% had good outcome with mRS (0-2) at 3-month follow-up compared to $35.51 \%$ in CT group.

Conclusion: The current standard neuroimaging in acute ischemic stroke patients is CT and CTA brain. Using MRI over CT scan for acute ischemic stroke may improve clinical outcomes for the subgroup of patients who have an unclear diagnosis and who have higher risk of complications with MT. Even though MRI and MRA take longer period to acquire, patient's clinical outcome was better in MRI group in comparison to CT group and was comparable to that of the five major endovascular trials.
\end{abstract}

Keywords: MRI brain, CT brain, Acute stroke, Mechanical thrombectomy

\section{Background}

Stroke is a major cause of mortality and morbidity worldwide. Ischemic stroke is the most common subtype of stroke accounting for around $80 \%$ of all stroke cases [1]. Ischemic stroke treatment includes recanalization using

\footnotetext{
*Correspondence: researchudr@gmail.com

tShivam Tiwari: Co-First author

${ }^{1}$ Department of Neurology, Pacific Medical College and Hospital, Pacific Medical University, Bhilon ka Bedla, Udaipur, Rajasthan 313001, India Full list of author information is available at the end of the article
}

either intravenous/intra-arterial thrombolysis, mechanical thrombectomy or sometime both. According to recent trials Mechanical thrombectomy has been proved to be effective treatment for ischemic stroke up to $24 \mathrm{~h}$ of onset of stroke [2].

In clinical practice, imaging has a big role in distinguishing between ischemia and intracerebral hemorrhage. Computed Tomography Scan (CT scan) and Magnetic Resonance Imaging (MRI) are the two imaging options for stroke cases. MRI has several 
advantages over CT Scan to examine the brain parenchyma in patients with acute ischemic stroke, such as detecting symptomatic ischemic lesions within few minutes of stroke onset, excluding stroke mimics, detecting ischemic lesions in the brain stem, distinguishing newly symptomatic from chronic ischemic lesions and establishing prognosis. This is true especially for the large "malignant" perfusion patterns at early timepoints, for which the risks of treatment may outweigh the benefits [1]. However, MRI has disadvantages, such as more acquisition time, contraindications with certain cardiac pacemaker, patients with severe claustrophobia and difficulty in monitoring of patients inside the MRI suite. The main reason many centers do not use MRI for routine stroke protocol is the time required to perform a complete examination, which can exceed $30 \mathrm{~min}$. This fact has limited the use of MRI as routine imaging protocol to screen for acute ischemic stroke patients for mechanical thrombectomy [3].

The most important MRI technique for selecting acute ischemic stroke patients for reperfusion therapy is Diffusion-weighted MR imaging (DWI). DWI has emerged as the most sensitive (88 to $100 \%$ ) and specific (95 to 100\%) imaging technique for acute infarct even at very early timepoints after symptom onset [4]. DWI can detect relatively small cortical lesions and deep or sub cortical lesions including those in the brain stem or cerebellum areas which are often poorly visualized with standard MRI sequences and CT scan [5].

MR angiography (MRA) is a vascular imaging technique which provides an essential piece of information in the initial assessment of acute stroke patients and in the evaluation of the outcome of reperfusion therapies. In fact, location and extension of the vascular occlusion provides valuable information in predicting response to intravenous thrombolysis and, therefore, for selecting appropriate candidates for intra-arterial thrombectomy [6]. MRA is commonly performed in combination with brain MRI in the setting of acute stroke to guide therapeutic decision making $[3,7]$. In nutshell CT-based protocols are easily available, time efficient, relatively free of motion artifacts and patient cooperation but MRI offers better infarct assessment, saving contrast use, radiation safety, better detection of posterior fossa infarct and micro bleeds. Additional Perfusion studies in CT-based protocol may address extent of infarct and oligemia much better though has inherent complexities in routine performance and interpretation.

The objective of this study is comparison of functional outcome in CT-based versus MRI-based evaluation of functional outcome in acute ischemic stroke.

\section{Methods}

This study is a prospective gathered in house registry (PMU/085/09/2018-09/2020) consisting of all patients with acute ischemic stroke at our Comprehensive stroke center. Participants were 152 patients admitted from September 2, 2018 to September 2, 2020 with acute ischemic stroke who underwent mechanical thrombectomy treatment. Patients with a clinical diagnosis of acute stroke were evaluated using National Institutes of Health Stroke Scale (NIHSS), modified Rankin scale (mRS) and were randomized into MRI brain-based protocol or CT-based protocol consecutively.

The regional review board has approved the use of human subjects for this study. All participants had given written informed consent. This Study did not require further approval as it used existing standard imaging, such as CT scan and MRI. There was no new drug or invasive procedure used in this study outside the standard existing recommendation.

\section{Patient inclusion and exclusion criteria}

Patients aged $18-80$ years with acute ischemic stroke and symptomatic anterior proximal large vessel occlusion on CTA/MRA/DSA with NIHSS of at least 2 points within $6 \mathrm{~h}$ of stroke onset with informed consent was included in the study. A specific exclusion criterion for intended mechanical thrombectomy is concurrent myocardial infarction or severe infection (endocarditis or sepsis), uncontrollable hypertension defined as systolic blood pressure $>185 \mathrm{mmHg}$ or diastolic pressure $>110 \mathrm{mmHg}$, Life expectancy of less than 90 days before stroke onset, pregnant or lactating women, known severe allergy to radiographic contrast medium and improvement of NIHSS score $>4$ in less than $1 \mathrm{~h}$. CT or MRI evidence of significant mass effect with midline shift, CT or MRI showing more than $1 / 3$ of MCA territory infarct, CT or MRI evidence of intracranial hemorrhage (ICH), Subarachnoid Hemorrhage (SAH), Aneurysm or Cerebral arteriovenous malformations (CAVMs).

Fast imaging techniques such as echo-planar imaging (EPI), Propeller (GE)/ BLADE (Siemens) and turbo spin echo has significantly reduced the timing of MRI scan without compromising the image quality. The purpose of this study was to establish the feasibility of a fast MR protocol that can be obtained in $\approx 13 \mathrm{~min}$.

In MRI on a $1.5 \mathrm{~T}$ (Siemens Essenza) MR system, we followed a protocol of MRI consuming less time taken with imaging protocol including diffusion-weighted imaging (DWI), EPI fluid attenuation inversion recovery imaging (FLAIR), EPI-gradient recalled echo (GRE), MRA brain \& neck (TOF). We removed the sagittal T2, coronal FLAIR and axial $\mathrm{T} 1$ and axial $\mathrm{T} 2$ sequences from the fast MR protocol for acute stroke to reduce 
timing as the above mentioned sequences does not affect the diagnosis of the stroke. If there is stroke mimic-like condition than we add these sequences for further additional information.

In addition to the new sequences which consume lesser amount of time, we have changed the certain parameters of the sequences. We have reduced the phase resolution from 80 to 70 in axial FLAIR and SWI sequences avoiding the $500 \mathrm{~B}$ value images and only acquired the 0 and $1000 \mathrm{~B}$ value images in DWI this further reduced the timings of the MRI scan. We have also changed the slice thickness of TOF angiography neck and brain from 0.6 to 0.8 and reduced phase FOV from 100 to $80-85$ (vary according the patient) and also reduced the base resolution of angiography from 320 to 256 and phase resolution to 70 to 63 reduce the scan time dramatically and when this MR scan compared with the MR scan obtained by the sequences with normal parameters (provided by factory settings of the machine), No significant difference in the image quality was shown.

Detailed parameters of sequence timings are shifting and preparation time- $2 \mathrm{~min}$, localizer- $20 \mathrm{~s}$, DWI Axial $1 \mathrm{~min} 10 \mathrm{~s}$, SWI Axial $1 \mathrm{~min} 27 \mathrm{~s}$, FLAIR Axial 2 min $5 \mathrm{~s}$, TOF MRA Brain 2 min $50 \mathrm{~s}$, Localizer Neck $15 \mathrm{~s}$ and TOF MRA Neck 2 min $50 \mathrm{~s}$. The time taken for the post processing of TOF angiography is negligible as after acquiring the TOF raw data we can obtain 3D image reconstruction in just $10 \mathrm{~s}$ each for brain and neck angiography. Total duration of the scan time is $12 \mathrm{~min}$ and $57 \mathrm{~s}$.

The 13-min time is an estimate calculated based on all steps required to complete the image acquisition. It should be noted that acquisition time may vary on a case by case basis depending on factors, such as patient cooperation and technical difficulties.

All the CT scan head and CT angiography were performed on the 128 slice dual source CT scanner of Somatom definition by Siemens.

When, we see the only scan timing, which is less than a minute, but few additional time consuming process which are also necessary for scan. The total, calculated time of CT scanning with reconstruction is approximately 10 min which is less than the time taken for MR stroke protocol. It does not include the timing of contrast filling in the injector as it is already prepared before patient shift.

The Various details of the timing for CT scan are shifting and positioning $2 \mathrm{~min}$, Topogram and planning $20 \mathrm{~s}$, CT head scan time $18 \mathrm{~s}$, Reconstruction time $2 \min 30 \mathrm{~s}$, Plain Angio scan $6 \mathrm{~s}$, Contrast Bolus tracking $40 \mathrm{~s}$, Angio run contrast $20 \mathrm{~s}$, Angio reconstruction $3 \mathrm{Min}$, total time is $9 \mathrm{~min} 23 \mathrm{~s}$ (Fig. 1).
We performed Modified Rankin Scale (MRS) at admission, and at 3 months. Patients who refused to sign the informed consent, non cooperative or non fit for MRI or CT scan for the study, were excluded before randomization. To achieve the objective the hypothesis is framed which is further tested (Table 2). There is no significant difference on outcome based on CT and MRI scan after 3 months.

\section{Statistical analysis}

The CT and MRI groups were compared using the Mann-Whitney $U$ test for quantitative variables and the $X^{2}$ test for qualitative variables. Factors with a significant $(P<0.10)$ association with favorable outcome in analysis-that is, age, hypertension, hyperlipidemia ischemic heart disease, National Institutes of Health Stroke Scale score, diabetes mellitus, history of previous stroke. For two groups CT-based versus MRI-based outcome prior to mechanical thrombectomy, and imaging modality (MRI versus CT) - were candidates for the model. Reported $P$ values were two-sided, with values $<0.05$ indicative of a significant difference. All tests were twosided. The statistical analysis was performed using SPSS (Version 25.0; IBM,)

\section{Results}

Total 152 patients were included in the study with mean age of 54.6 (range 22-80) years with male and female being 102 and 50, respectively. Both groups CT protocol performed and MRI protocol performed had 76 patients each. Mean NIHSS on admission was $17(4-30)$ and $16(4-30)$ and at discharge was $7(2-23)$ and $6(2-22)$ in CT-based and MRI-based groups, respectively. Results showed that $44.07 \%$ and $43.42 \%$ of the patients had hypertension, smoker $30.26 \%$ and $34.21 \%$, diabetes mellitus $27.63 \%$ and $38.15 \%$ hyperlipidemia $55.26 \%$ and $50 \%$, ischemic heart disease $15.78 \%$ and $13.15 \%$, tobacco chewing $60.52 \%$ and $57.89 \%$, alcohol $28.94 \%$ and $23.68 \%$, history of previous stroke $19.73 \%$ and $18.42 \%$, as a risk factor of stroke in CT and MRI group, respectively. There was no statistical significant difference in as tandem lesion is $6.57 \%$ and $7.89 \%$ in CT and MRI group, respectively, ICAD is $18.42 \%$ and $19.73 \%$ in CT and MRI group, respectively, $\mathrm{T}$ occlusion is $26.3 \%$ and $30.26 \%$ in $\mathrm{CT}$ and MRI group, respectively, and IV rt-PA is $28.95 \%$ and $27.63 \%$ in CT and MRI group, respectively, before enrollment in the study (Table 1). Both groups were age, sex matched and risk factors were not statistically different. Average stroke onset to door time was $227 \mathrm{~min}$ (range 45-360) and 229(47-360), door to needle time was 110 (range 54-300) and 125 (range 60-310) and stroke to groin puncture time was 342 min (range 120600) and $350 \mathrm{~min}$ (range 126-610) in CT and MRI group, 


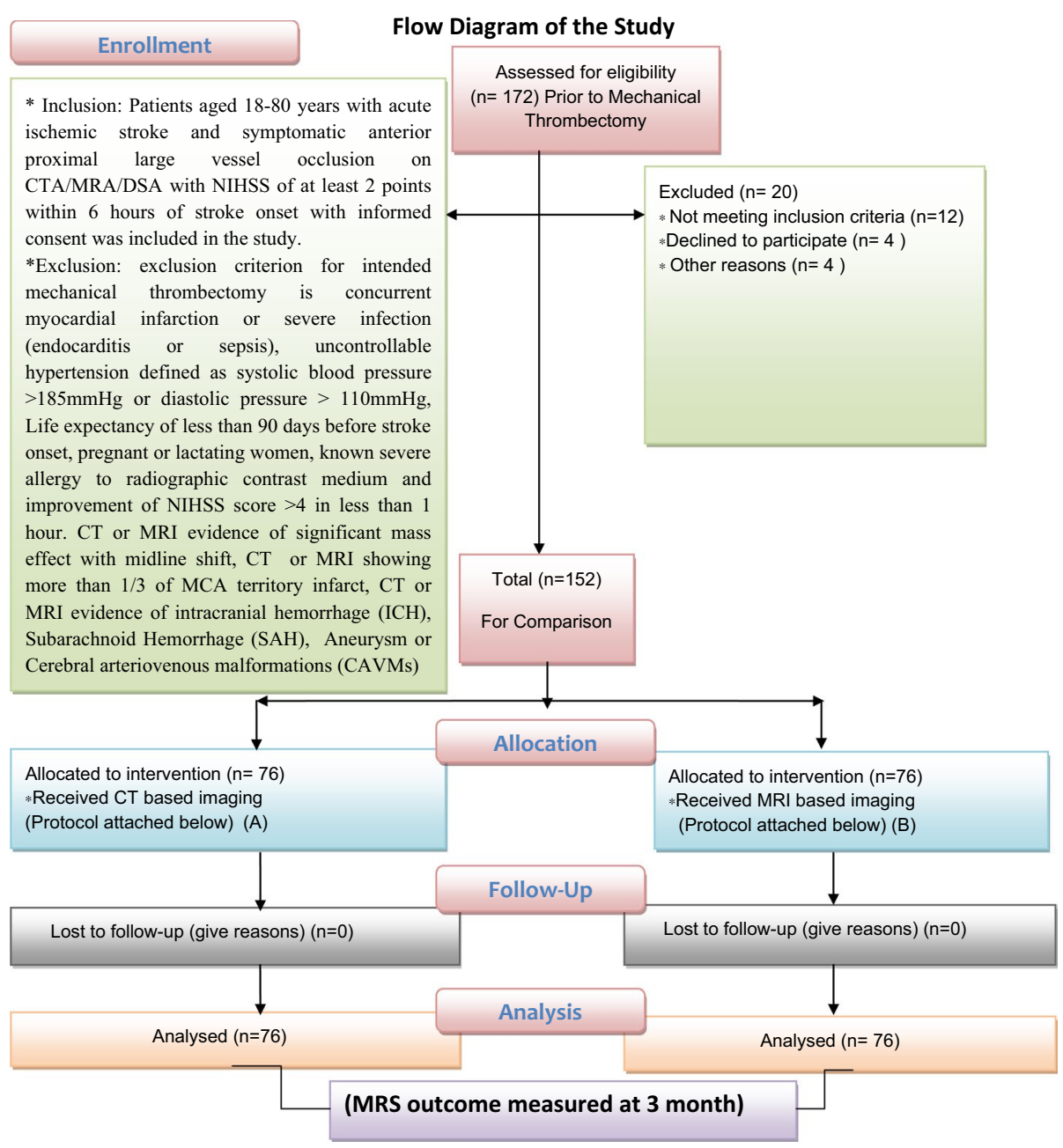

CT Timing Protocol Used (A)

\begin{tabular}{|l|l|l|}
\hline S.No. & Process/Planning & Timing \\
\hline 1. & Shifting \& positioning & $2 \mathrm{~min}$ \\
\hline 2. & $\begin{array}{l}\text { Topogram and } \\
\text { planning }\end{array}$ & $20 \mathrm{sec}$ \\
\hline 3. & CT head scan time & $18 \mathrm{sec}$ \\
\hline 4. & Reconstruction time & $2 \mathrm{~min} 30 \mathrm{sec}$ \\
\hline 5. & Plain Angio scan & $6 \mathrm{sec}$ \\
\hline 6. & Contrast Bolus tracking & $40 \mathrm{sec}$ \\
\hline 7. & Angio run contrast & $20 \mathrm{sec}$ \\
\hline 8 & Angio reconstruction & $3 \mathrm{Min}$ \\
\hline 9. & Total & $\mathbf{9 ~ m i n ~ 2 3 ~ s e c ~}$ \\
\hline
\end{tabular}

MRI Timing Protocol Used (B)

\begin{tabular}{|c|c|c|}
\hline S.No. & Sequence/Planning & Timing \\
\hline 1. & $\begin{array}{c}\text { Shifting \& Preparation } \\
\text { Time }\end{array}$ & 2 min \\
\hline 2. & Localizer & $1 \mathrm{~min} 10 \mathrm{sec}$ \\
\hline 3. & DWI Axial & $1 \mathrm{~min} 27 \mathrm{sec}$ \\
\hline 4. & SWI Axial & $2 \mathrm{~min} 5 \mathrm{sec}$ \\
\hline 5. & FLAIR Axial & $2 \mathrm{~min} 50 \mathrm{sec}$ \\
\hline 6. & TOF MRA Brain & $15 \mathrm{sec}$ \\
\hline 7. & Localizer Neck & $2 \mathrm{~min} 50 \mathrm{sec}$ \\
\hline 8. & TOF MRA Neck & $\mathbf{1 2} \mathbf{m i n} \mathbf{5 7} \mathbf{~ s e c}$ \\
\hline & TOTAL & \\
\hline
\end{tabular}

Fig. 1 Flow diagram of the study. CT and MRI timing protocols used in the study. The CT and MRI groups were compared using the MannWhitney $U$ test for quantitative variables and the $x^{2}$ test for qualitative variables 
Table 1 Baseline characteristics and outcome parameters according to the proposed criteria of the CT and MRI

\begin{tabular}{llll}
\hline ( $\boldsymbol{n}=\mathbf{1 5 2 )}$ & $\mathbf{C T}$ & MRI & $\boldsymbol{p}$ values \\
\hline Characteristics & $N=76 n(\%)$ & $N=76 n(\%)$ & 0.34 \\
Age mean (range) (years) & $51.4(22-76)$ & $57.8(23-80)$ & 0.23 \\
Sex (male) \% & $50(65.78 \%)$ & $52(68.42 \%)$ & 0.31 \\
Smoker & $23(30.26 \%)$ & $26(34.21 \%)$ & 0.42 \\
Diabetes mellitus & $21(27.63 \%)$ & $29(38.15 \%)$ & 0.54 \\
Hypertension & $34(44.07 \%)$ & $33(43.42 \%)$ & 0.64 \\
hyperlipidemia & $42(55.26 \%)$ & $38(50 \%)$ & 0.65 \\
Ischemic heart disease & $12(15.78 \%)$ & $10(13.15 \%)$ & 0.66 \\
Tobacco chewing & $46(60.52 \%)$ & $44(57.89 \%)$ & 0.67 \\
Alcohol & $22(28.94 \%)$ & $18(23.68 \%)$ & 0.73 \\
History of previous stroke & $15(19.73 \%)$ & $14(18.42 \%)$ & 0.76 \\
IV rtPA & $26(28.95 \%)$ & $23(27.63 \%)$ & 0.56 \\
Tandem lesions & $5(6.57 \%)$ & $6(7.89 \%)$ & 0.48 \\
ICAD & $14(18.42 \%)$ & $15(19.73 \%)$ & 0.62 \\
T-occlusion & $20(26.31 \%)$ & $23(30.26 \%)$ & 0.54 \\
Baseline NIHSS-median & $17(4-30)$ & $16(4-30)$ & 0.86 \\
(IQR) & & & \\
mRS at 90 days-median & $5(3-6)(n=76)$ & $1(0-2)(n=76)$ & $0.006^{*}$ \\
(IQR) & & &
\end{tabular}

Data are presented as $n / N(\%)$ or median (interquartile range). $C T$ computed tomography, MRI magnetic resonance imaging, NIHSS National Institutes of Health Stroke Scale, ICAD Intracranial atherosclerotic disease

* Significant

respectively (Table 2). In the MRI group 65.5\% patients had good outcome (MRS between 0 and 2) at 3-month follow-up in comparison to $35.51 \%$ in CT group. In this study recanalization rate were comparable in both $\mathrm{CT}$ and MRI group.TICI $2 \mathrm{~b}-3$ in $92 \%$ in MRI group and $90 \%$ in $\mathrm{CT}$ group. There is statistically significant difference in hemorrhagic transformation after the procedure in $\mathrm{CT}$ (27.63\%) and MRI (2.63\%) group, respectively. Hypothesis testing result suggest that MRI protocol group had better modified Rankin scale-based outcome compared to $\mathrm{CT}$ scan protocol group. Imaging modality was not significantly associated with functional outcome in the multivariable analysis. (Tables 3, 4, Figs. 2, 3.)

\section{Discussion}

We compared our study's CT and MRI evaluation protocol groups and also compared with big trials using CTbased protocols, such as MR CLEAN, ESCAPE, EXTEND IA, SWIFT PRIME, and REVASCAT. We can see that the stroke onset to door time in our institution is longer, with a difference 23 min with CT group and 25 min with MRI group compared to MR CLEAN, 58 min with CT group and 60 min with MRI group compared to ESCAPE, 37 min with CT group and 39 min with MRI group compared to SWIFT PRIME, and $4 \mathrm{~min}$ with CT group and 6 min with MRI group compared to REVASCAT. This means that we need improvement in several points such as educating people in our city for stroke awareness and also improve the quality service of the picking up patients using ambulance [8]

This means that despite the use of MRI Brain as protocol of neuroimaging in acute stroke ischemic patients, our door to groin puncture time is not significantly different from CT-based studies [8].

The result of follow-up of our study reveals good outcome (MRS 0-2), in 65.5\% of MRI group patients compared to $35.51 \%$ in CT group patients, while in other CT-based studies, good outcome was present in $33 \%$ in MR CLEAN [9], 51\% in ESCAPE [10], 75\% in EXTEND IA [11], 59\% in SWIFT PRIME, and 44\% in REVASCAT [12]. In follow-up outcome (MRS at 3 months), our studies is only inferior to EXTEND IA studies. This means that in reality our treatment is slightly delayed due to MRI examination. We had better or non inferior patients outcome compared to our own and other major studies published (MR CLEAN, ESCAPE, EXTEND IA, SWIFT PRIME, and REVASCAT) [8].

Probably MRI brain offers better selection for mechanical thrombectomy due to better assessment of extent of infarct, thereby increasing safer outcome of patients.

Table 2 Comparison data between our study and five major mechanical thrombectomy trials

\begin{tabular}{|c|c|c|c|c|c|c|c|}
\hline & \multicolumn{2}{|c|}{$\begin{array}{l}\text { PMCH } \\
\text { Total } N=152\end{array}$} & \multirow[t]{2}{*}{$\begin{array}{l}\text { MR CLEAN } \\
N=233\end{array}$} & \multirow[t]{2}{*}{$\begin{array}{l}\text { ESCAPE } \\
N=165\end{array}$} & \multirow[t]{2}{*}{$\begin{array}{l}\text { EXTEND IA } \\
N=35\end{array}$} & \multirow[t]{2}{*}{$\begin{array}{l}\text { SWIFT PRIME } \\
N=98\end{array}$} & \multirow[t]{2}{*}{$\begin{array}{l}\text { REVASCAT } \\
N=103\end{array}$} \\
\hline & $\begin{array}{l}\mathrm{CT} \\
N=76\end{array}$ & $\begin{array}{l}\text { MRI } \\
N=76\end{array}$ & & & & & \\
\hline Imaging modalities & CT, CTA & MRI, MRA & CT, CTA, MRA perfusion & $\mathrm{CT}, \mathrm{CTA}$ & CT, CTA, CT Perfusion & CT, CTA, CT Perfusion & $C T, C T A, M R I$ \\
\hline Stroke onset to Door time & 227 & 229 & 204 & 169 & - & 190 & 223 \\
\hline Door to Groin Puncture time & 110 & 125 & - & 90 & 113 & 95 & 109 \\
\hline $\begin{array}{l}\text { Stroke onset to groin puncture } \\
\text { time }\end{array}$ & 342 & 350 & 260 & - & 210 & 224 & 269 \\
\hline
\end{tabular}


Table 3 Comparison of MRS (\%) scores at 3 months between our study and other clinical trials

\begin{tabular}{|c|c|c|c|c|c|c|}
\hline MRS & MRI & MR CLEAN & ESCAPE & EXTENDIA & SWIFT PRIME & REVASCT \\
\hline 0 & 18.42 & 3 & 14 & 25 & 17 & 7 \\
\hline 1 & 27.63 & 9 & 20 & 25 & 25 & 18 \\
\hline 2 & 19.73 & 21 & 17 & 20 & 17 & 19 \\
\hline 3 & 11.84 & 19 & 16 & 17 & 12 & 18 \\
\hline 4 & 7.89 & 22 & 13 & 3 & 15 & 8 \\
\hline 5 & 10.52 & 6 & 6 & 0 & 3 & 12 \\
\hline 6 & 3.94 & 21 & 10 & 9 & 9 & 18 \\
\hline Good (MRS 0-2) & 65.78 & 33 & 51 & 70 & 59 & 44 \\
\hline Bad (MRS 3-6) & 34.19 & 68 & 45 & 29 & 39 & 56 \\
\hline
\end{tabular}

Table 4 Patients MRS Score after 3 months, evaluated through $\mathrm{CT}$ and MRI

\begin{tabular}{lcc}
\hline MRS & PMCH & \\
\cline { 2 - 3 } & CT \% & MRI\% \\
\hline 0 & 7.89 & 18.42 \\
1 & 11.84 & 27.63 \\
2 & 15.78 & 19.73 \\
3 & 14.47 & 11.84 \\
4 & 25 & 7.89 \\
5 & 15.78 & 10.52 \\
6 & 9.21 & 3.94 \\
Good (MRS 0-2) & 35.51 & 65.78 \\
Bad (MRS 3-6) & 64.46 & 34.19 \\
\hline
\end{tabular}

PCMH Pacific Medical College and Hospital
Similar results were shown in patients undergoing IVT in extended window period patients in MRI-based protocols [13]. Few previous studies compared CT and MRI-based protocol in acute ischemic stroke prior to endovascular therapy with no overall increase in better outcome with MRI though reduced risk of symptomatic intra cerebral hemorrhage was reported $[14,15]$.

\section{Conclusion}

The current standard neuroimaging in acute ischemic stroke patients is CT and CTA brain. Using MRI over CT scan for acute ischemic stroke may improve clinical outcomes for the subgroup of patients who have an unclear prognosis, diagnosis and who have higher risk with mechanical thrombectomy. It appears that clearer and

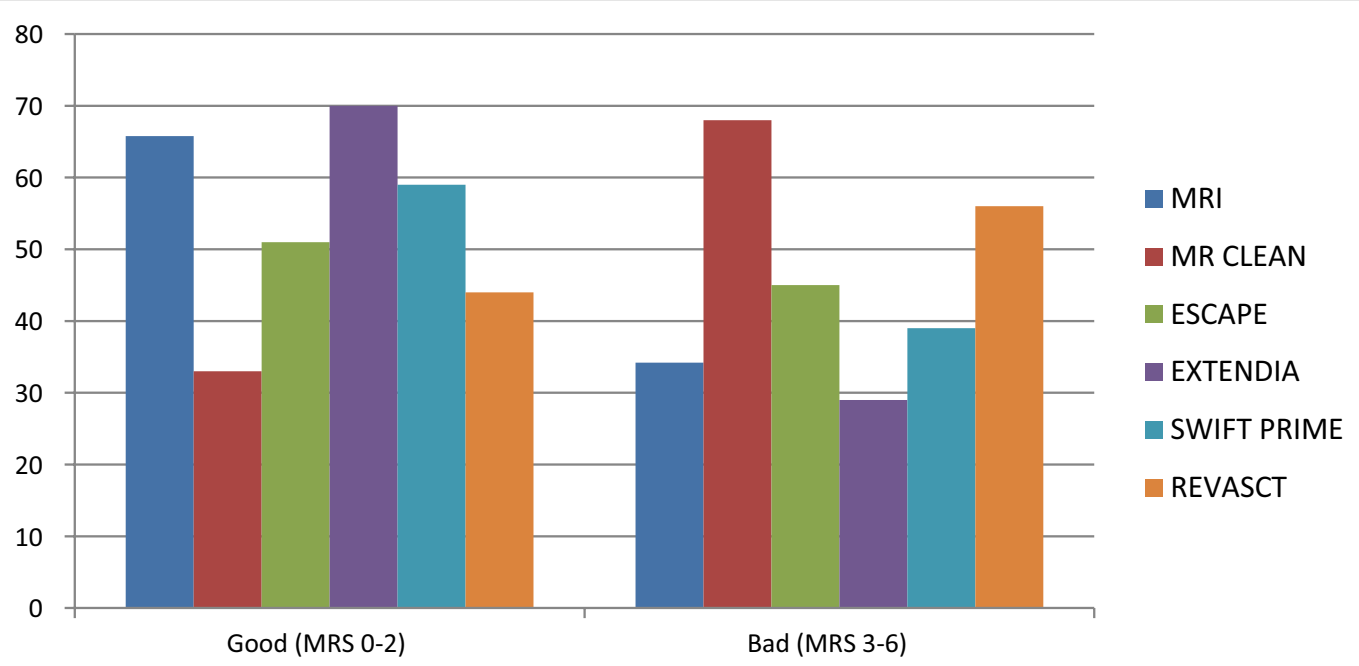

Fig. 2 Comparison of outcomes with PMCH MRI trial and other five clinical trials. PCMH Pacific Medical College and Hospital 
MRS Outcome after 3 Months

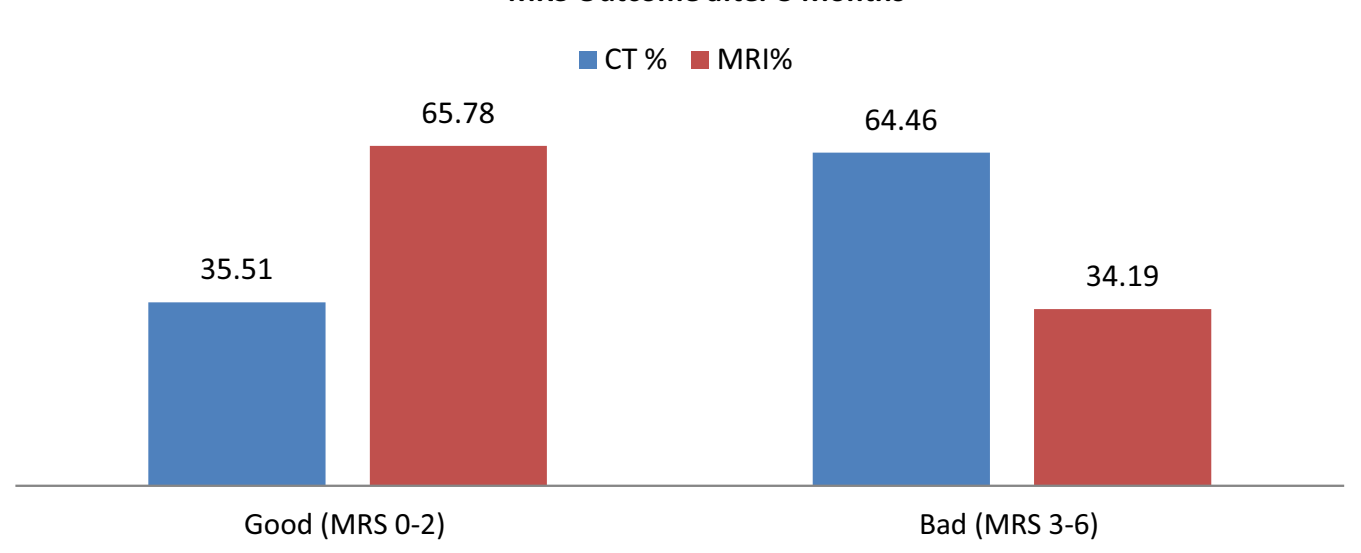

Fig. 3 Percentage of patients MRS scores after 3 months, evaluated through CT and MRI

earlier visualization of bigger infarct on MRI and MRA compared to CT and CTA might save patient from futile recanalization, hemorrhagic transformation, poor outcome and extra cost despite slightly longer MRI acquision time especially in the absence of perfusion imaging.

\section{Abbreviations}

CT: Computed tomography; CTA: Computed tomography angiography; MRA: Magnetic resonance angiography; MRI: Magnetic resonance imaging; DWI: Diffusion-weighted imaging; mRS: Modified rankin; GCS: Glasgow Coma Scale; NIHSS: National Institutes of Health stroke scale; MT: Mechanical thrombectomy; DSA: Digital subtraction angiography; ICH: Intracranial hemorrhage; SAH: Subarachnoid hemorrhage; CAVMs: Aneurysm or cerebral arteriovenous malformations; FLAIR: EPI fluid attenuation inversion recovery imaging; GRE: EPI-gradient recalled echo; PMCH: Pacific Medical College and Hospital.

\section{Acknowledgements}

The authors acknowledge the Pacific Medical University and Hospital, for providing facilities and support during the study, the authors acknowledge subjects for their participation and cooperation in this study. We sincerely thank to Dr. Shivoham Singh for Statistical analysis and we thank to Yogya Tiwari for revising and English language editing in the manuscript.

\section{Authors' contributions}

AV, MV, ST, LBY, NM, and KV implemented the study. KV performed Imaging modalities and protocol. ST and LBY, KV, DJ, SD, data collection. AV and ST, LBY performed data analyses and data interpretation. AV, ST, LBY, and MV drafted the initial manuscript. AV, NM, DJ, SD, KV, ST, LBY, and MV critically reviewed the manuscript and provided inputs. AV, ST, and LBY contributed equally. All authors read and approved the manuscript.

\section{Funding}

This research received no specific grant from any funding agency in the public, commercial, or not-for-profit sectors.

\section{Availability of data and materials}

All authors confirm the availability of data and materials.

\section{Declarations}

Ethics approval and consent to participate

The Institutional ethics committee Pacific Medical University has approved the use of human subjects for this study. With approval Dated 6th of September
2018 with the reference number (PMU/085/09/2018-09/2020) consisting of all patients with acute ischemic stroke at our Comprehensive stroke center. All participants had given written, informed consent.

\section{Consent for publication}

Not applicable.

\section{Competing interests}

The authors declare that they have no competing interests.

\section{Author details}

${ }^{1}$ Department of Neurology, Pacific Medical College and Hospital, Pacific Medical University, Bhilon ka Bedla, Udaipur, Rajasthan 313001, India. ${ }^{2}$ Department of Neurosurgery, Pacific Medical College and Hospital, Pacific Medical University, Bhilon ka Bedla, Udaipur, Rajasthan 313001, India. ${ }^{3}$ Department of Radiology, Pacific Medical College and Hospital, Pacific Medical University, Bhilon ka Bedla, Udaipur, Rajasthan 313001, India. ${ }^{4}$ Department of Reproductive Medicine and Research, Pacific Medical College and Hospital, Pacific Medical University, Bhilon ka Bedla, Udaipur, Rajasthan 313001, India.

Received: 10 August 2021 Accepted: 4 February 2022

Published online: 22 February 2022

\section{References}

1. Donkor ES. Stroke in the 21st Century: a snapshot of the burden, epidemiology, and quality of life. Stroke Res Treat. 2018;2018:3238165. https:// doi.org/10.1155/2018/3238165.

2. Nogueira RG, Jadhav AP, Haussen DC, et al. Thrombectomy 6 to 24 hours after stroke with a mismatch between deficit and infarct. N Engl J Med. 2018:378(1):11-21. https://doi.org/10.1056/NEJMoa1706442.

3. Jauch EC, Saver JL, Adams HP Jr, Bruno A, Connors JJ, Demaerschalk $B M$, et al. Guidelines for the early management of patients with acute ischemic stroke: a guideline for healthcare professionals from the American Heart Association/American Stroke Association. Stroke. 2013;44(3):870-947.

4. Latchaw RE, Alberts MJ, Lev MH, Connors JJ, Harbaugh RE, Higashida $R T$, et al. Recommendations for imaging of acute ischemic stroke: a scientific statement from the American Heart Association. Stroke. 2009;40(11):3646-78.

5. Lee LJ, Kidwell CS, Alger J, Starkman S, Saver JL. Impact on stroke subtype diagnosis of early diffusion-weighted magnetic resonance imaging and magnetic resonance angiography. Stroke. 2000;31(5):1081-9.

6. Rubiera M, Ribo M, Delgado-Mederos R, Santamarina E, Delgado P, Montaner J, et al. Tandem internal carotid artery/middle cerebral artery 
occlusion: an independent predictor of poor outcome after systemic thrombolysis. Stroke. 2006;37(9):2301-5.

7. Vert C, Parra-Fariñas C, Rovira À. MR imaging in hyperacute ischemic stroke. Eur J Radiol. 2017;96:125-32.

8. Powers WJ, Rabinstein AA, Ackerson T, Adeoye OM, Bambakidis NC, Becker K, Biller J, et al. 2018 Guidelines for the early management of patients with acute ischemic stroke: a guideline for healthcare professionals from the American Heart Association/American Stroke Association. Stroke. 2018;49(3):e46-99.

9. Fransen PS, Beumer D, Berkhemer OA, Van Den Berg LA, Lingsma H, van der Lugt A, et al. MR CLEAN, a multicenter randomized clinical trial of endovascular treatment for acute ischemic stroke in the Netherlands: study protocol for a randomized controlled trial. Trials. 2014;15(1):1-1.

10. Arch AE, Weisman DC, Coca S, Nystrom KV, Wira CR III, Schindler JL. Missed ischemic stroke diagnosis in the emergency department by emergency medicine and neurology services. Stroke. 2016;47(3):668-73.

11. Bhaskar S, Stanwell P, Cordato D, Attia J, Levi C. Reperfusion therapy in acute ischemic stroke: dawn of a new era? BMC Neurol. 2018;18(1):1-26.

12. Mokin M, Rojas H, Levy El. Randomized trials of endovascular therapy for stroke_-impact on stroke care. Nat Rev Neurol. 2016:12(2):86.

13. Köhrmann M, Jüttler E, Fiebach JB, Huttner HB, Siebert S, Schwark C, et al. MRI versus CT-based thrombolysis treatment within and beyond the $3 \mathrm{~h}$ time window after stroke onset: a cohort study. Lancet Neurol. 2006;5(8):661-7.

14. Provost C, Soudant M, Legrand L, Ben Hassen W, Xie Y, Soize S, et al. Magnetic resonance imaging or computed tomography before treatment in acute ischemic stroke: effect on workflow and functional outcome. Stroke. 2019;50(3):659-64.

15. Kim JT, Cho BH, Choi KH, Park MS, Kim BJ, Park JM, et al. Magnetic resonance imaging versus computed tomography angiography based selection for endovascular therapy in patients with acute ischemic stroke. Stroke. 2019;50(2):365-72.

\section{Publisher's Note}

Springer Nature remains neutral with regard to jurisdictional claims in published maps and institutional affiliations.

\section{Submit your manuscript to a SpringerOpen ${ }^{\circ}$ journal and benefit from:}

- Convenient online submission

- Rigorous peer review

- Open access: articles freely available online

- High visibility within the field

- Retaining the copyright to your article 